\title{
Beliefs Regarding Fundamental Value and Optimal Investing *
}

\author{
Bradford Cornell ${ }^{\dagger}$ \\ Jakša Cvitanić $\ddagger$ \\ Levon Goukasian §
}

June 4, 2008

\begin{abstract}
We derive optimal portfolio weights for an investor who has specific beliefs regarding the distribution of a stock price at a future time. For example, a fundamental investor will want to take advantage of the information his analysis provides when constructing a portfolio. In this regard, we examine the optimal weights for models in which the investor believes that there is a range in which the price is likely to remain. Under such circumstances, the optimal strategy is to take significant long/short positions as the price nears its lower/upper boundary. The risk, expected stock return and the optimal investment strategy are thus dependent on the stock price relative to the boundaries.
\end{abstract}

Keywords: Investor beliefs, mispricing, optimal portfolios, range reversion, risk premium. JEL Classification: G11

${ }^{*}$ The previous version of this paper was circulated under the title "Optimal investing with perceived mispricing". J.C.'s research supported in part by NSF grants DMS 04-03575 and DMS 06-31298, and through the Programme "GUEST" of the National Foundation For Science, Higher Education and Technological Development of the Republic of Croatia. We are solely responsible for any remaining errors, and the opinions, findings and conclusions or suggestions in this article do not necessarily reflect anyone's opinions but the authors'.

†Caltech, Humanities and Social Sciences, M/C 228-77, 1200 E. California Blvd. Pasadena, CA 91125. Ph: (626) 833-9978. E-mail: bcornell@hss.caltech.edu

${ }_{\ddagger}^{\ddagger}$ Caltech, Humanities and Social Sciences, M/C 228-77, 1200 E. California Blvd. Pasadena, CA 91125. Ph: (626) 395-1784. E-mail: cvitanic@hss.caltech.edu.

$\S$ Business Division, Pepperdine University, Malibu, CA 90263. Ph: (310) 506-4425. E-mail: Levon.Goukasian@Pepperdine.edu. 


\section{Introduction}

Actively managed mutual funds are an essential part of the financial industry. From the standpoint of an active investor, there is a fundamental paradox with respect to the application of equilibrium asset pricing models. To model the relation between risk and expected returns, asset pricing models employ highly restrictive assumptions. The most stringent of these assumptions, employed by most standard models, is that investors have homogeneous beliefs defined over investments opportunities that are either stationary or whose variation is determined by stationary stochastic processes. Given these assumptions, equilibrium asset pricing equations can be derived that provide a precise definition of risk and its relation to asset expected returns ${ }^{1}$. The paradox is that the reason for being an active investor is the belief that some combination of asset price volatility, nonstationarity, incomplete and asymmetric information, and heterogeneous information processing capabilities, lead to inefficient pricing that can be exploited ${ }^{2}$. This implies that, from the standpoint of such active investors, standard asset pricing models must be incorrect. However, if they are not applicable, how is the active investor to measure risk and assess the trade-off between risk and expected return? This problem has been recognized in various contexts by numerous professional investors. For example, as reported by Penman (2007), famed investor Warren Buffett puts the matter this way: "The CAPM says that if the price of a stock drops more than the market, it has a high beta: It's high risk. But if the price goes down because the market is mispricing the stock relative to other stocks, then the stock is not necessarily high risk: The chance of making an abnormal return has increased, and paying attention to fundamentals makes the investor more secure, not less secure. ${ }^{3}$ " In a similar vein, Morningstar (2004), a leading provider of investment analysis, rejects asset pricing based measures of risk, stating that, "In deciding the rate to discount future cash flows, we ignore stock-price volatility (which drives most estimates of beta) because we welcome volatility if it offers opportunities to buy a stock at a discount to its fair value. Instead, we focus on the fundamental risks facing a company's business. Ideally, we'd like our discount rates to reflect the risk of permanent capital loss to the investor. When assigning a cost of equity to a stock, our analysts score a company in the following areas:

Financial leverage - The lower the debt, the better.

Cyclicality - The less cyclical the firm, the better.

Size - We penalize very small firms.

\footnotetext{
${ }^{1}$ See Cochrane (2005) for example, for a detailed theory.

${ }^{2}$ Friedman (1953) argues that security prices reflect fundamental values. Otherwise, if securities are mispriced, as a result of irrational investors' behavior, rational investors will take advantage of the mispricing, and therefore push prices to their fundamentals. Other researchers, on the other hand, argue that security prices may persistently diverge from their fundamentals. Shiller (1984), De Long et al. (1990) argue that when the transaction costs exceed potential profits then the prices may not converge to their fundamentals. Campbell and Shiller (2001) show that deviations from the fundamentals have provided valuable forecasting information for future stock prices. When the Price/Earnings ratio has been above its historical mean, stock prices tended to fall. Likewise, when the Dividends/Price ratio has been above its mean, stock prices tended to rise.

${ }^{3}$ This is consistent with the Fama and French (1992) observation that Book-to-Market (B/M) ratios are positively correlated with subsequent stock returns, a relation that is known as the book-to-market effect. That is, value stocks - the stocks with lower $\mathrm{B} / \mathrm{M}$ ratios yield higher returns compared to growth stocks stocks with higher $\mathrm{B} / \mathrm{M}$ ratios.
} 
Free cash flow - The higher as a percentage of sales and the more sustainable, the better."

Both Buffet and Morningstar are getting at the same basic point. If markets are sometimes inefficient, then it makes little sense to rely on measures of risk derived from equilibrium asset pricing models. At a minimum, such models fail to take account of the fact that when the price of an asset is low, relative to the fundamentals as assessed by the investor, then the risk will be less. Given the focus on construction of equilibrium models, financial economists have not spent much time developing techniques for assessing risk in inefficient markets. This is no doubt due in part to the fact the problem faced by an active investor of measuring risk in an "inefficient" market cannot be unambiguously defined. It depends on the precise nature of the inefficiency that the investor believes exists. Early efforts to integrate perceived superior information into the context of the CAPM floundered on this problem. Despite the inherent ambiguities, modern derivative pricing techniques can provide active investors with useful tools for assessing risk in markets which they believe reflect meaningful mispricing ${ }^{4}$. With that goal in mind, and in the spirit of the quotes from Warren Buffett and Morningstar, this paper examines a situation in which the active investor believes that he can place bounds on the variation of the price of certain specific stocks.

More specifically, we consider two related types of market inefficiency from the standpoint of the active investor. In the first instance, the investor believes that he can determine fundamental bounds on the value of a stock during the time interval, $[0, T]$. If the market price penetrates either of those boundaries, it reverts back toward the boundary ${ }^{5}$. The speed of reversion is varied in our analysis. We refer to this first case as range reversion or $\mathrm{RR}^{6}$.

In the second, related case, the active investor believes that as of time 0 he can place strict bounds on the distribution of a stock price at time T. As a result, rather than being lognormal, as in the Black-Scholes framework, the time $\mathrm{T}$ distribution of the stock price is truncated at the boundaries. More generally, we allow the investor to specify any continuous distribution for the price at time $T$. This case is referred to as range distribution, or $\mathrm{RD}^{7}$. In both cases, it is intuitively clear that the active investors will perceive that both the risk and expected return of the stock will be a function of the current price. ${ }^{8}$

The fact that risk and return vary with price, from the standpoint of the active investor, is contrary to standard models such as the $\mathrm{CAPM}^{9}$ or the Black-Scholes-Merton option

\footnotetext{
${ }^{4}$ Under the assumption of existence of market frictions and imperfections, Basak and Croitoru (2000) show that mispricing can be sustainable in general equilibrium.

${ }^{5}$ As Campbell and Shiller (2001) state it, the mean reversion in the valuation ratios and its forecasting ability for future prices are not new concepts. Mean reversion in fundamentals has been frequently discussed as a forecasting tool for price movements over the last century.

${ }^{6}$ Our model reflects the assumption that stock prices can diverge from their fundamentals for prolonged periods. Theorizing this has been a challenge for financial economists. Campbell and Shiller (2001) review the proposed solutions to this problem. Our model is consistent with the Campbell and Shiller (2001) observation that prices rather than fundamentals do most of the adjustment in bringing the ratios back towards their long-run equilibrium levels. Thus, our model is consistent with their argument that valuation ratios (fundamental bounds in our case) can be used to predict stock price changes.

${ }^{7}$ Liu and Longstaff (2004) study a related problem. In their setting, an investor has perfect knowledge about the future value of the security at time $T$, and thus faces an arbitrage opportunity related to a security, which is modeled as a Brownian Bridge process.

${ }^{8}$ This result is consistent with the 3 -factor pricing model of Fama and French (see Fama and French (1993)) in which the factors HML or SMB are functions of the price of the security.

${ }^{9}$ see Sharpe $(1964,1970)$, Lintner $(1965 \mathrm{a}, 1965 \mathrm{~b})$, and Mossin (1966).
} 
pricing mode ${ }^{10}$ in which risk and expected return are not correlated with the price of a security. Furthermore, the distinction between the standard models and the models that we analyze becomes more pronounced as the price approaches a boundary. Naturally, the relation of the price to the boundary also affects the optimal holdings of the active investor. We analyze those holdings in two contexts, in which the investor invests in a risky stock, an index and a risk-free asset. The first context is a mean-variance framework in which the portfolio holdings are set at time 0 and cannot be altered. The second is a dynamic optimization context in which the portfolio holdings can be adjusted continuously. ${ }^{11}$

Our analysis is presented as follows. We begin with a formal mathematical discussion of both cases. To simplify the presentations, the results are given without proofs which are available from the authors. In situations where there are no closed form solutions to the problems, we provide Monte Carlo simulations. We report on numerical computations of optimal portfolios in the models, useful for estimating the economic magnitude of the effects we consider. In particular, we examine how optimal holdings of the active investor, and his perceived risk and expected return vary as a function of price. We calculate the dependence of betas, and expectations and variances of relative returns on the stock price relative to the boundaries. Finally, we compare the performance of the models under consideration with traditional models and analyze the welfare gain/loss of the active investor from exploiting his fundamental information. We finish with some conclusions and possible extensions.

\section{RR Model: "Range Reversion"}

In the range reversion model the active investor believes he can determine an approximate lower bound $L_{t}$ and an approximate upper bound $U_{t}$ of a risky asset with price $S_{t}$. We call asset $S$, the stock. The investor believes that the price will revert back into the range $\left[L_{t}, U_{t}\right]$ with a certain speed over a given time interval $[0, T]$. We allow $L_{t}$ and $U_{t}$ to vary with time. This type of modeling is similar to traditional mean reversion models, except in those models the stock price reverts back to a single value. Consequently, mean reversion models are a special case of our model in which $L_{t}=U_{t}$.

For Monte Carlo computations, it is more convenient to work with log-values. Thus, we write the range reversion model as follows. Denote

$$
\tilde{L}_{t}=\log L_{t}, \quad \tilde{U}_{t}=\log U_{t}, \quad Y_{t}=\log S_{t} .
$$

In this framework, the log-asset price $Y_{t}$ is given by the Stochastic Differential Equation $(\mathrm{SDE})$

$$
d Y_{t}=\left[\mu_{t}-\sigma_{t}^{2} / 2+n_{L} \max \left\{0, \tilde{L}_{t}-Y_{t}\right\}-n_{U} \max \left\{0, Y_{t}-\tilde{U}_{t}\right\}\right] d t+\sigma_{t} d B_{t}
$$

\footnotetext{
${ }^{10}$ See Black and Scholes (1973), and Merton (1973).

${ }^{11}$ Black and Litterman (see Black and Litterman (1990)) were one of the first to use investors' subjective views along with CAPM as a benchmark in portfolio management context. Their model uses a Bayesian approach to combine the subjective views of an investor regarding the expected returns of assets with the market equilibrium expected returns (via the prior distribution) to form a new estimate of expected returns. The resulting new set of returns (the posterior distribution) leads to portfolios with more stable weights and thus results in a better asset allocation.
} 
Here, $B_{t}$ is a one-dimensional standard Brownian Motion process, and $n_{L}$ and $n_{U}$ are the "speeds of reversion" from the lower and upper bounds respectively. If the stock price $S$ is below $L$ (log-stock price $Y$ is below $\tilde{L}$ ) the term containing $n_{L}$ pushes up the price back towards $L$. Similarly if $S$ is above $U$ it reverts back toward U at speed $n_{U}$. Setting $L=-\infty$, $U=\infty$, gives the standard generalized Black-Scholes-Merton model, henceforth BSM model. In the computations we will take $\mu, \sigma$ to be deterministic.

\subsection{Optimal investing in the RR model}

In this section we compare optimal investments of a pasive investor who accepts BSM model with an active investor who uses a RR model. Consider a BSM model for the stock price

$$
\hat{S}_{t}=\hat{S}_{0} e^{\left(\hat{\mu}-\hat{\sigma}^{2} / 2\right) t+\hat{\sigma} B_{t}}
$$

Also assume that there exists a market index I, modeled as a geometric Brownian Motion process:

$$
I_{t}=I_{0} e^{\left(\mu_{I}-\sigma_{I}^{2} / 2\right) t+\sigma_{I}\left[\rho B_{t}+\sqrt{1-\rho^{2}} W_{t}\right]}
$$

where $W$ is a Brownian Motion process, independent of $B$. Moreover, assume that there is a risk-free asset with constant interest rate $r \geq 0$.

We assume that both the BSM investor and the RR investor have the same beliefs regarding the index $I$ and the risk-free asset. They differ in their beliefs about the dynamics of $S$.

For the given time horizon $T>0$, we assume that $\sigma, \mu$ in the $\mathrm{RR}$ model (2.1) are constants and that

$$
\tilde{L}_{t}=\tilde{L}-r(T-t), \quad \tilde{U}_{t}=\tilde{U}-r(T-t)
$$

where $\tilde{L}, \tilde{U}$ are constants. In other words, the likely range for the final stock price $S_{T}$ is $\left[L_{T}, U_{T}\right]$, and the likely range for the value of $S_{t}$ for $t<T$ is $\left[L_{T} e^{-r(T-t)}, U_{T} e^{-r(T-t)}\right]$.

As the measure of risk we compute the beta of asset $S^{12}$, defined by

$$
\beta=\frac{\operatorname{Cov}\left[I_{T} / I_{0}, S_{T} / S_{0}\right]}{\operatorname{Var}\left[I_{T} / I_{0}\right]} .
$$

To solve the portfolio optimization problem, we assume that the investors have logarithmic preferences in order to simplify the calculations. Given this framework, we compute optimal investment weights for both a static, Markowitz Mean-Variance portfolio, and for a dynamic, Merton Log-optimal portfolio.

More specifically, denote the value of the investor's portfolio at time $\mathrm{T}$ by $X_{T}$. For the Markowitz Mean-Variance setting, we consider the maximization of

$$
E\left[\frac{X_{T}}{X_{0} e^{r T}}-1-\frac{\gamma}{2}\left(\frac{X_{T}}{X_{0} e^{r T}}-1\right)^{2}\right]
$$

In particular, with the risk aversion parameter $\gamma$ equal to one, this approximates $\log \left(X_{T} / X_{0}\right)=$ $\log \left(1+\left(\frac{X_{T}}{X_{0} e^{r T}}-1\right)\right)$. For the Merton case, we maximize $E\left[\log \left(X_{T}\right)\right]$.

\footnotetext{
${ }^{12}$ We only consider here partial equilibrium, in which investors take prices as given and cannot influence them; thus, it is not a priori clear that beta is the right measure of risk in this model. We will consider full equilibrium implications of heterogeneous beliefs on the range of prices in future work.
} 


\subsubsection{Optimal mean-variance investment}

To compare our results with those of the standard model, we denote by $\pi=\left(\pi_{S}, \pi_{I}\right)$ the optimal weight proportions in assets $S$ and $I$ respectively, for the RR model. The optimal weights for the BSM investor are denoted by, $\hat{\pi}=\left(\hat{\pi}_{S}, \hat{\pi}_{I}\right)$. We place a superscript $M, \pi^{M}$ for the static, Markowitz case, and no superscript for the dynamic, Merton case. Finally, in order to simplify notation, we define the quantities representing return in excess of the risk free rate,

$$
\bar{I}_{T}=I_{T}-I_{0} e^{r T}, \quad \bar{S}_{T}=S_{T}-S_{0} e^{r T} .
$$

Given this framework, we first derive formulas for optimal investment strategies, and later compare numerical results for the two models.

Proposition 2.1 For an investor maximizing expected value of the expression in (2.3), the optimal static portfolio proportions are given by

$$
\begin{aligned}
& \pi_{S}^{M}=\frac{2-\gamma}{\gamma} S_{0} e^{r T} \frac{E\left[\bar{S}_{T}\right] E\left[\bar{I}_{T}^{2}\right]-E\left[\bar{I}_{T} \bar{S}_{T}\right] E\left[\bar{I}_{T}\right]}{E\left[\bar{I}_{T}^{2}\right] E\left[\bar{S}_{T}^{2}\right]-\left(E\left[\bar{S}_{T} \bar{I}_{T}\right]\right)^{2}} \\
& \pi_{I}^{M}=\frac{2-\gamma}{\gamma} S_{0} e^{r T} \frac{E\left[\bar{I}_{T}\right] E\left[\bar{S}_{T}^{2}\right]-E\left[\bar{S}_{T} \bar{I}_{T}\right] E\left[\bar{S}_{T}\right]}{E\left[\bar{I}_{T}^{2}\right] E\left[\bar{S}_{T}^{2}\right]-\left(E\left[\bar{S}_{T} \bar{I}_{T}\right]\right)^{2}}
\end{aligned}
$$

and similarly for $\hat{\pi}_{S}^{M}$ and $\hat{\pi}_{I}^{M}$, which are just like the above expressions with $\hat{S}_{T}$ used instead of $\bar{S}_{T}$.

Proof: This is obtained by standard mean-variance optimization and the result is familiar: the optimal portfolio is proportional to the inverse of the variance-covariance matrix applied on the excess returns:

$$
\left\{\begin{array}{cc}
E\left[\bar{S}_{T}^{2}\right] & E\left[\bar{S}_{T} \bar{I}_{T}\right] \\
E\left[\bar{S}_{T} \bar{I}_{T}\right] & E\left[\bar{I}_{T}^{2}\right]
\end{array}\right\}^{-1}\left(E\left[\bar{S}_{T}\right], E\left[\bar{I}_{T}\right]\right)^{T r}
$$

The main reason why the optimal strategies will qualitatively differ between the RR and the BSM model is that in the BSM model the expected relative return is given by

$$
E\left[\hat{S}_{T} / S_{0}\right]=e^{\hat{\mu} T}
$$

which is independent of the current stock price. This is not the case for the RR model, where the expected return and the beta depend on the stock price relative to the boundaries. In the Comparative Statics subsection below, we provide numerical results which illustrate the impact of the stock price on risk and expected return. 


\subsubsection{Optimal dynamic investment}

Next, we consider vectors of proportions $\pi, \hat{\pi}$ that are optimal for the investor who can continuously rebalance his portfolio (without transaction costs or other frictions).

For the RR model with constant volatility $\sigma$, in the notation

$$
d S_{t}=\mu_{t}^{S} S_{t} d t+\sigma_{t}^{S} S_{t} d B_{t}
$$

we have

$$
\begin{gathered}
\sigma_{t}^{S}=\sigma, \\
\mu_{t}^{S}=\mu_{t}+n_{L} \max \left\{0, \tilde{L}_{t}-\log S_{t}\right\}-n_{U} \max \left\{0, \log S_{t}-\tilde{U}_{t}\right\}
\end{gathered}
$$

Introduce the volatility matrix,

$$
\Sigma_{t}=\left\{\begin{array}{cc}
\sigma_{t}^{S} & 0 \\
\rho \sigma_{I} & \sqrt{1-\rho^{2}} \sigma_{I}
\end{array}\right\}
$$

and the vector of excess expected return rates,

$$
R_{t}=\left\{\mu_{t}^{S}-r, \mu_{I}-r\right\}
$$

(Actually, for neither model does $\Sigma$ depend on $t$.) Then, from the classical Merton's problem (see Merton $(1969,1971)$ ), we know that the optimal vector of proportions $\pi$ of wealth to be held in $S$ and $I$, for an investor with logarithmic utility, is given by

$$
\pi_{t}=\left(\Sigma_{t} \Sigma_{t}^{T r}\right)^{-1} R_{t}
$$

In the same fashion, we can solve for the optimal $\hat{\pi}_{t}$ in the BSM model with $\mu^{S}, \sigma^{S}$ replaced by $\hat{\mu}, \hat{\sigma}$. We use (2.7) to compute optimal dynamic portfolios. Again, the main difference between the two models is that the parameters entering the computation in (2.7) are independent of the stock price for the BSM model, while this is not the case for the RR model.

\subsubsection{Comparative Statics}

To assess the economic significance of the difference between the RR and BSM models we use the following benchmark parameters, which approximate those observed for individual common stocks and a hypothetical market index:

$$
\begin{gathered}
T=1, r=0.03, \quad I_{0}=1, \quad S_{0}=1, X_{0}=1, \quad \mu=0.08, \quad \sigma=0.3, \quad \mu_{I}=0.05, \quad \sigma_{I}=0.25, \quad \rho=0.2, \\
\tilde{U}=\log S_{0}+\mu T+2 \sigma \sqrt{T}, \quad \tilde{L}=\log S_{0}+\mu T-2 \sigma \sqrt{T}, \quad \gamma=1 .
\end{gathered}
$$

We choose $\hat{\mu}, \hat{\sigma}$ so that the mean and the variance of the stock price at time $T$ are the same in the two models, for $S_{0}=\hat{S}_{0}=1$. That is, we choose $\hat{\mu}$ and $\hat{\sigma}$ so that

$$
\begin{gathered}
E\left[S_{T}\right]=E\left[\hat{S}_{T}\right]=e^{\hat{\mu} T}, \\
E\left[S_{T}^{2}\right]=E\left[\hat{S}_{T}^{2}\right]=e^{\left(2 \hat{\mu}+\hat{\sigma}^{2}\right) T} .
\end{gathered}
$$


In the calculations, we keep $\hat{\mu}$ and $\hat{\sigma}$ fixed at the initial level, corresponding to $S_{0}=1$, but allow $S_{0}$ to vary. The interpretation of this procedure is as follows. Suppose the stock price is low today. For the BSM investor (since BSM is a stationary model) this does not change the long-run mean $\hat{\mu}$ and volatility $\hat{\sigma}$ (and thus the risk-premium on the stock). Consequently, the optimal investment weights will not change with the stock price. For the $\mathrm{RR}$ investor, however, if the stock price is close to what the active investor believes is its approximate lower bound, the risk premium should be higher (because the expected returns would be higher and beta lower) than predicted by BSM model, and the optimal weight should be greater. Tables 1 and 2 present the results of the calculations comparing the RR model with the BSM model. ${ }^{13}$ Panel A of Table 1 shows that the investment weights for the RR investor change markedly as the stock price approaches either of the bounds. When the stock is near the bottom boundary the $\mathrm{RR}$ investor greatly increases his relative holding in the stock and the reverse is true for the upper boundary ${ }^{14}$. In order to compensate for this behavior, the RR investor also takes more extreme positions in the index than the BSM investor, but not as extreme as in the stock, because the index has unlimited range in the model $^{15}$. Panel B of Table 1 shows the Merton's optimal dynamic portfolio weights for the Log-investors of the RR type and the BSM type. The quantitative results are even more dramatic than those for the mean-variance calculation, but the qualitative behavior is the same. Table 2 shows how the weights change with the speed of range reversion. For low speeds, less than 0.1, the weights are very close to BSM weights and do not vary much with the initial stock prices. As the RR speeds rise, the investment proportions vary more with stock prices. For example, for the speed of $n_{L}=n_{U}=1$, the optimal allocation to the stock is about $93 \%$ as the stock price approaches its lower bound, and is $-74 \%$ as the stock price gets closer to its upper bound. This shows the dependence of the investor's risk-taking on the speed of reversion from the bounds. However, after the value of 100 of the reversion speed, further variation in the weights is limited.

\section{RD Model: Modeling return distribution at a future time}

We now analyze a class of models in which the active investor believes he has superior information regarding the future stock price distribution, at a fixed future time $T$. For example,

\footnotetext{
${ }^{13}$ The portfolio weights for the RR model are actually expected values of the portfolio weights at time $t=T / 2$. We do this because at time $t=0$ the stock price is inside the believed bounds, and not much interesting happens.

${ }^{14}$ This may help explain why fund managers may want to hold less-diversified portfolios. A fund manager that has superior skills or information which allow him to estimate boundaries, may decide to hold a more concentrated portfolios. Coval and Moskowitz $(1999,2001)$ show that mutual funds have strong preferences for investing in local firms where they might have informational advantages. Our analysis is consistent with those results.

${ }^{15}$ These findings may be used to explain the investment strategies of corporate executives. Under the assumption that executives are more knowledgeable about their company's future prospects, they can better assess the "closeness" of their company's stock price to fundamental bounds and make investment decisions based on that assessment. For example, if an executive believes his firm's stock is close to the lower bound (or is undervalued relative to the assessed fundamentals), he will hold more shares and thus, be under-diversified.
} 
this is consistent with the investor who believes that the stock price will be constrained to be in the vicinity of its fundamental value in the long run. We refer to this scenario as the RD model ${ }^{16}$.

More specifically, given a future time $T$, let $S_{T}$ be a random variable which represents the active investor's beliefs regarding the distribution of the stock price at time $T$. We assume that $S_{T}$ is of the form

$$
S_{T}=f\left(B_{T}^{Q}\right)
$$

where $f$ is a deterministic function and $B^{Q}$ is a Brownian Motion process, but under a probability $Q$ which is risk-neutral from the point of view of the active investor. From his point of view, Brownian Motion process $B$ under the actual physical probability is such that

$$
B^{Q}(t)=B(t)+\theta^{Q} t
$$

where the risk premium $\theta^{Q}$ perceived by the investor is assumed to be constant. From the investor's point of view, the stock price is the expected value of its discounted future value under probability $Q$. That is, we have

$$
S_{t}=E_{t}^{Q}\left[e^{-r(T-t)} f\left(B_{T}^{Q}\right)\right] .
$$

Denote by $N(\cdot)$ the standard normal cumulative distribution function. We have the following useful result:

Proposition 3.1 (i) For an arbitrary continuous distribution function $F$, if we set

$$
S_{T}=F^{-1}\left(N\left(B_{T} / \sqrt{T}\right)\right)
$$

then $S_{T}$ will have $F$ as its distribution.

(ii) Furthermore, from the investor's point of view, $S_{t}=V\left(t, B_{t}^{Q}\right)$, where the function $V$ is a solution to the following PDE:

$$
\partial_{t} V+\frac{1}{2} \partial_{x x} V-r V=0
$$

with the boundary condition

$$
V(T, x)=f(x), \forall x
$$

Proof: Part (i) is straightforward. Part (ii) is a direct consequence of the Feynman-Kac theorem and (3.1).

The focus in the next subsection is on the investor's modeling the stock price at a fixed future time, and his calibrating the risk premium $\theta^{Q}$ to the current stock price using (3.1) with $t=0$. Put differently, because the active investor believes in a different distribution than the BSM investor, he also believes in different risk premium, hence in different riskneutral probability.

\footnotetext{
${ }^{16}$ Liu and Longstaff (2004) study the investment decisions of an active investor who faces an arbitrage opportunity regarding the future value of a security about which the investor has perfect foresight.
} 


\subsection{BSMCT Model}

To make the analysis tractable, we study a special case of RD models, a model for which the $\log$-price $Y_{T}=\log S_{T}$ has a conditionally truncated normal distribution. More precisely, the distribution of $Y=Y_{T}$ is the distribution of the normal random variable with mean

$$
m=\log S_{0}+\left(\mu-\sigma^{2} / 2\right) T
$$

and variance

$$
\tilde{\sigma}^{2}=\sigma^{2} T
$$

conditional on taking values in the interval with endpoints

$$
\tilde{L}=\log L, \quad \tilde{U}=\log U .
$$

We call this model BSMCT (for Black-Scholes-Merton Conditionally Truncated). It is known that the distribution function of $Y$ is then given by

$$
F_{Y}(y)=\frac{N\left(\frac{y-m}{\tilde{\sigma}}\right)-N\left(\frac{\tilde{L}-m}{\tilde{\sigma}}\right)}{N\left(\frac{\tilde{U}-m}{\tilde{\sigma}}\right)-N\left(\frac{\tilde{L}-m}{\tilde{\sigma}}\right)} .
$$

Computing $F_{Y}^{-1}$ from the preceding, we see from (3.2) that the corresponding model for the stock price at time $T$ is

$$
\log \left(S_{T}\right)=m+\tilde{\sigma} N^{-1}\left\{N\left(\frac{\tilde{L}-m}{\tilde{\sigma}}\right)+N\left(\frac{B_{T}^{Q}-\theta^{Q} T}{\sqrt{T}}\right)\left[N\left(\frac{\tilde{U}-m}{\tilde{\sigma}}\right)-N\left(\frac{\tilde{L}-m}{\tilde{\sigma}}\right)\right]\right\}
$$

Recall that $\theta^{Q}$ is not a free parameter - it has to be chosen so that

$$
S_{0}=E^{Q}\left[e^{-r T} S_{T}\right]
$$

Denoting

$$
U^{*}=\frac{\tilde{U}-m}{\tilde{\sigma}}, \quad L^{*}=\frac{\tilde{U}-m}{\tilde{\sigma}}
$$

the formulas needed for computations are based on the following proposition.

Proposition 3.2 In the BSMCT model, the stock price at time $T$ can be written as

$$
S_{T}=e^{m+\tilde{\sigma} N^{-1}}\left\{N\left(L^{*}\right)+N\left(\frac{B_{T}^{Q}-T \theta^{Q} Q}{\sqrt{T}}\right)\left[N\left(U^{*}\right)-N\left(L^{*}\right)\right]\right\}
$$

and the stock price for $t<T$ is given by $S_{t}=V\left(t, B_{t}^{Q}\right)$ where

$$
V(t, x)=e^{-r(T-t)} E^{Q}\left[e^{m+\tilde{\sigma} N^{-1}\left\{N\left(L^{*}\right)+N\left(\frac{x+B_{T}^{Q}-B_{t}^{Q}-\theta^{Q}(T-t)}{\sqrt{T-t}}\right)\left[N\left(U^{*}\right)-N\left(L^{*}\right)\right]\right\}}\right] .
$$

We also have

$$
V_{x}(0,0)=e^{-r T} E^{Q}\left[\frac{\tilde{\sigma}}{\sqrt{T}} S_{T} \frac{n\left(\frac{B_{T}^{Q}-T \theta^{Q}}{\sqrt{T}}\right)\left[N\left(U^{*}\right)-N\left(L^{*}\right)\right]}{n\left(\frac{\log S_{T}-m}{\tilde{\sigma}}\right)}\right]
$$


Proof: We get the expression for $S_{T}$ from (3.4), we get (3.5) from (3.1), and (3.6) from differentiating (3.5).

If we write,

$$
d S_{t}=S_{t}\left[\mu_{t}^{S} d t+\sigma_{t}^{S} d B_{t}\right]
$$

in the terminology of CAPM, we can define the "instantaneous beta" of asset $S$ by

$$
\beta_{S}(t)=\frac{\rho \sigma_{t}^{S}}{\sigma_{I}}
$$

where $\sigma_{I}$ is the volatility of the index. In order to compute the instantaneous beta of $S$ we need the following lemma, which follows directly from Ito's rule.

Lemma 3.1 In BSMCT model, we have

$$
\sigma_{t}^{S}=\frac{V_{x}\left(t, B_{t}^{Q}\right)}{S_{t}}
$$

\subsection{Optimal investing in the BSMCT model}

In this section, we compare optimal investments of an investor who uses a BSM model or an $\mathrm{RR}$ model, and an investor who uses a BSMCT model. The BSM model for $S$ is the same as in the previous section. As before, we also assume that both the BSM investor and the BSMCT investor have the same beliefs regarding the index $I$ and the risk-free asset, but they differ in their beliefs about $S$. Note that since $S_{T}$ takes values in $[L, U]$, the stock price values before $T$ are contained in the corresponding discounted interval $\left[L e^{-r(T-t)}, U e^{-r(T-t)}\right]$. We again assume that the investors have logarithmic utility and we compute optimal investment weights for a static, Markowitz mean-variance portfolio, and for a dynamic, Merton logoptimal portfolio. The qualitative behavior of the static portfolios is the same as for the dynamic portfolios, but they differ quantitatively - the latter are more variable than the former.

\subsubsection{Mean-Variance portfolio}

The formulas here are the same as in the RR case. From Proposition 2.1:

$$
\begin{aligned}
\pi_{S}^{M} & =\frac{2-\gamma}{\gamma} S_{0} e^{r T} \frac{E\left[\bar{S}_{T}\right] E\left[\bar{I}_{T}^{2}\right]-E\left[\bar{I}_{T} \bar{S}_{T}\right] E\left[\bar{I}_{T}\right]}{E\left[\bar{I}_{T}^{2}\right] E\left[\bar{S}_{T}^{2}\right]-\left(E\left[\bar{S}_{T} \bar{I}_{T}\right]\right)^{2}} \\
\pi_{I}^{M} & =\frac{2-\gamma}{\gamma} S_{0} e^{r T} \frac{E\left[\bar{I}_{T}\right] E\left[\bar{S}_{T}^{2}\right]-E\left[\bar{I}_{T} \bar{S}_{T}\right] E\left[\bar{S}_{T}\right]}{E\left[\bar{I}_{T}^{2}\right] E\left[\bar{S}_{T}^{2}\right]-\left(E\left[\bar{S}_{T} \bar{I}_{T}\right]\right)^{2}}
\end{aligned}
$$

and similarly for $\hat{\pi}_{S}^{M}$ and $\hat{\pi}_{I}^{M}$, which are just like the above expressions with $\overline{\hat{S}_{T}}$ used instead of $\bar{S}_{T}$. 


\subsubsection{Dynamic portfolios}

We know from proposition 3.2 that $S_{t}=V\left(t, B_{t}^{Q}\right)$ and also that

$$
d S_{t}=r S_{t} d t+V_{x}\left(t, B_{t}^{Q}\right) d B_{t}^{Q}
$$

It follows from this that

$$
d S_{t}=\mu_{t}^{S} S_{t} d t+\sigma_{t}^{S} S_{t} d B_{t}
$$

with

$$
\mu^{S}=r+\theta^{Q} V_{x} / S, \quad \sigma^{S}=V_{x} / S
$$

Introduce the volatility matrix,

$$
\Sigma=\left\{\begin{array}{cc}
\sigma^{S} & 0 \\
\rho \sigma_{I} & \sqrt{1-\rho^{2}} \sigma_{I}
\end{array}\right\}
$$

and the vector of excess returns,

$$
R=\left\{\mu^{S}-r, \mu_{I}-r\right\}
$$

Then, from the classical Merton problem, we know that the vector of optimal proportions, $\pi_{t}$, of wealth to be held in stock and in the index for an investor with logarithmic utility is given by

$$
\pi=\left(\Sigma \Sigma^{\prime}\right)^{-1} R
$$

Similarly, the optimal proportion, $\hat{\pi}$, in the Black-Scholes model is calculated by replacing $\mu^{S}, \sigma^{S}$ with $\hat{\mu}, \hat{\sigma}$ in the above formula (3.10). We use (3.10) to compute optimal dynamic portfolios.

\subsection{Comparative Statics}

In the numerical computations, we again use these benchmark parameters as in the previous section:

$T=1, r=0.03, I_{0}=1, S_{0}=1, X_{0}=1, \mu=0.08, \quad \sigma=0.3, \quad \mu_{I}=0.05, \quad \sigma_{I}=0.25, \quad \rho=0.2$,

$$
L=0.65, \quad U=2, \quad \gamma=1 .
$$

We choose $\hat{\mu}, \hat{\sigma}$ so that the mean and the variance of the stock at time $T$ are the same in the two models, for $S_{0}=\hat{S}_{0}=1$. That is, we choose $\hat{\mu}$ and $\hat{\sigma}$ so that

$$
\begin{gathered}
E\left[S_{T}\right]=E\left[\hat{S}_{T}\right]=e^{\hat{\mu} T}, \\
E\left[S_{T}^{2}\right]=E\left[\hat{S}_{T}^{2}\right]=e^{\left(2 \hat{\mu}+\hat{\sigma}^{2}\right) T} .
\end{gathered}
$$

As before, we keep $\hat{\mu}$ and $\hat{\sigma}$ fixed at this level corresponding to $S_{0}=1$, but vary initial stock price $S_{0}$,in this case by varying risk premium $\theta^{Q}$.

We consider three different cases of the BSMCT model. In the first, we fix the value $m$ of (3.3) at the value corresponding to $S_{0}=1$. This represents the case in which the investor has a fixed belief about the distribution of the final stock price $S_{T}$, independent of the current 
stock price $S_{0}$. In the second case, which we call " $m$ floating", we will let $m$ change with $S_{0}$. This reflects an active investor whose belief about $S_{T}$ changes with the current value of $S_{0}$, and is, in this sense, close to the BSM investor, except that the distribution of the log-price for him is a conditionally truncated normal distribution. In the third model, $m$ is fixed, but with $L=0$ and $U=\infty$. That is, there are no bounds on the distribution of $S_{T}$, as in the BSM model, but the investor does not adapt his beliefs about $S_{T}$ as the value of $S_{0}$ changes, unlike in the BSM model.

Table 3 shows how the risk premium $\theta^{Q}$ changes with the stock price for those three models. The table shows that overall, the premium varies least as a function of the stock price in the model with no bounds. However, near the initial stock price the $m$ floating version shows less variation. The first model with fixed beliefs shows the greatest variation in the premiums as a function of the stock price.

Panel A of Table 4 presents expected returns $E\left[S_{T} / S_{0}\right]$ for all four non-standard models. The m-fixed models with and without the bounds cannot be distinguished, indicating that the bounds are not restrictive with respect to the expected returns. These are the models in which the investor fixes the distribution of $S_{T}$ in his decision making, which is independent of the initial value $S_{0}$ of stock price. The expected returns in the RR case are least variable in the initial stock price because the bounds in that model are soft bounds.

Panel B of Table 4 presents the variances $\operatorname{Var}\left[S_{T} / S_{0}\right]$ of returns. Again, the two m-fixed models are similar, and have larger variance changes than the RR and $\mathrm{m}$-floating models. This is partly because we divide by $S_{0}$, and the numerator does not change in the m-fixed models.

Panel A of Table 5 presents the results for the static betas as defined in (2.2), which mirror the results for the variances across the different models. On the other hand, the instantaneous betas in panel B of Table 5, as defined in (3.7), are quite different from the static betas. In all three BSCT models: the instantaneous betas are low when the stock price is close to the lower and upper bounds, and higher in the middle, but they are always lower than the stationary beta in the BSM model. This shows that because of his perceived fundamental information the active investor will always interpret stocks about which he has information as less risky. The greater variation in beta as a function of the stock price is due to the fact that this is a local beta, which does not take into account possible future changes of the stock price dynamics, and the fact that the local volatility is lower when closer to the bounds. The instantaneous beta of the RR model is not presented, but it is the same as the stationary BSM beta, since the volatility is fixed in the RR model.

Panels A, B, and C of Table 6 present optimal Markowitz weights in the stock and the index for the $m$-fixed, $m$-floating and the unbounded BSMCT models, respectively. Table 7 shows the stock investment weight as a function of the stock price for all four models. Once again, the two $m$-fixed cases, with and without bounds are very similar. The RR case shows the least variation, and the $m$-floating case is the most variable at the boundaries of the range. 


\section{Utility Gains and Losses across models}

In this section we compare the performances of static Markowitz investment strategies that follow the proposed models. The benchmark for comparison in the first case is the situation in which the investor follows the RR model, taken as the true model of stock price movements. The benchmark for comparison in the second case is the situation in which the investor follows the BSM model, taken as the true model of stock price movements. In both cases, we compare the certainty equivalents of the utility of terminal wealth of the investor that follows a "wrong" model (the perceived stock price dynamics are different from the true dynamics), and the investor that follows the benchmark.

The wealth-process of the investor at time $T$ is given by

$$
X_{T}\left(S, \delta_{S}, \delta_{I}\right)=\delta_{I} I_{T}+\delta_{S} S_{T}+\left(X_{0}-\delta_{I} I_{0}-\delta_{S} S_{0}\right) e^{r T}
$$

where $\delta_{I}$ and $\delta_{S}$ are computed using (2.4):

$$
\begin{aligned}
& \delta_{S} S_{0} / X_{0}=\frac{2-\gamma}{\gamma} S_{0} e^{r T} \frac{E\left[\bar{S}_{T}\right] E\left[\bar{I}_{T}^{2}\right]-E\left[\bar{I}_{T} \bar{S}_{T}\right] E\left[\bar{I}_{T}\right]}{E\left[\bar{I}_{T}^{2}\right] E\left[\bar{S}_{T}^{2}\right]-\left(E\left[\bar{S}_{T} \bar{I}_{T}\right]\right)^{2}} \\
& \delta_{I} I_{0} / X_{0}=\frac{2-\gamma}{\gamma} S_{0} e^{r T} \frac{E\left[\bar{I}_{T}\right] E\left[\bar{S}_{T}^{2}\right]-E\left[\bar{I}_{T} \bar{S}_{T}\right] E\left[\bar{S}_{T}\right]}{E\left[\bar{I}_{T}^{2}\right] E\left[\bar{S}_{T}^{2}\right]-\left(E\left[\bar{S}_{T} \bar{I}_{T}\right]\right)^{2}} .
\end{aligned}
$$

Using the dynamics of $S$ and $I$, and the above formulas with $\gamma=1$, we can compute the expected utility of the investor

$$
u\left(S, \delta_{S}, \delta_{I}\right):=E\left[\frac{X_{T}\left(S, \delta_{S}, \delta_{I}\right)}{X_{0} e^{r T}}-1-\frac{1}{2}\left(\frac{X_{T}\left(S, \delta_{S}, \delta_{I}\right)}{X_{0} e^{r T}}-1\right)^{2}\right]
$$

In this context, we study the performances of our models by examining the certainty equivalents $(\mathrm{CE})$ of the models and comparing them to the ones of the benchmark case. We do this analysis for the case of Markowitz' static portfolios. Define the following ratios in the Markowitz' case:

(i)

$$
\frac{C E\left(S^{R R}, \delta_{S}^{B S M}, \delta_{I}^{B S M}\right)}{C E\left(S^{R R}, \delta_{S}^{R R}, \delta_{I}^{R R}\right)} .
$$

Here, the denominator represents the $\mathrm{CE}$ of an investor who believes the stock price follows the RR model and the stock price does follow the RR model. The numerator is the CE of the investor, who believes the stock price follows the BSM model, whereas the stock follows the RR model. Thus, this ratio measures the relative gain/loss in CE of a BSM investor in case he is wrong about the model. We study the welfare gain/loss of an investor by computing this ratio in the RR-model, and also in the three cases of the BSMCT model: $m$-fixed, $m$-floating, and $L=0, U=\infty$.

(ii)

$$
\frac{C E\left(S^{B S M}, \delta_{S}^{R R}, \delta_{I}^{R R}\right)}{C E\left(S^{B S M}, \delta_{S}^{B S M}, \delta_{I}^{B S M}\right)} .
$$

In this case, in the denominator we have the $\mathrm{CE}$ of an investor who believes the stock follows the BSM model and the stock does follow the BSM model. In the numerator we have the 
$\mathrm{CE}$ of the investor who believes that the stock price follows the RR model, whereas, in reality, the stock price follows the BSM model. Thus, the above ratio measures the relative gain/loss in $\mathrm{CE}$ of an $\mathrm{RR}$ investor in case he is wrong about the model. We do a similar exercise replacing RR model with our other non-standard models.

As in previous sections, we normalize the parameters so that the means and the variances are the same at $S_{0}=1$ for the models under consideration. We see from Table 8 that if the non-BSM investor is wrong, the relative loss in CE gets larger as we move away from $S_{0}=1$, up to a point, and it is far bigger for values of $S$ that are higher than one. In other words, if the BSM model is correct, the non-BSM investor will bear a loss for using a wrong model, especially if the stock price is high and he believes it cannot go much higher, so that it is optimal for him to take a short position in the stock. The losses on the low end of the stock price are also not insignificant - when the stock price is about half the starting value of $\$ 1$, the CE of the non-BSM investor can take values anywhere from $50 \%$ of the BSM investor's profit to a loss of five times as high as BSM investor's profit, depending on the model.

On the other hand, CE losses for the BSM investor who is wrong about the model are less extreme for most values of the stock prices, and don't vary as dramatically with those values. Nevertheless, they can be very high, and sometimes even higher than the relative $\mathrm{CE}$ losses of the non-BSM investor when he is wrong. In particular, this happens in a range of somewhat high values of $S$, for which the CE value of the BSM investor can represent a loss which is three times as high as non-BSM investor's profit, depending on the model. In other words, when the stock still has some room to move up, but not that much, and it is optimal to sell it short, the investor who incorrectly believes in the BSM model can experience significant losses.

Overall, the most interesting message of this section is that, even though in most cases the non-standard investor is exposed to a higher mean-variance loss if he is wrong about the model than the loss of the standard investor when he is wrong, there is a range of stock prices for which the standard investor is in fact at a risk of higher utility loss if he is wrong about the model of the stock price movements.

\section{Conclusions and Extensions}

From the standpoint of active investors, standard equilibrium asset pricing models cannot be correct. As Warren Buffett notes, the very reason for being an active investor is the belief that certain securities are not valued appropriately. If that is the case, active investors cannot rely on the risk and expected return results derived from equilibrium models. This leads to the question of how the active investor should assess the risk and expected return of an individual security. There is no general answer to that question. It depends on the type of misvaluation that the active investor believes exists. In this paper, we analyze a basic form of mispricing. In particular, we assume that, perhaps via the application of fundamental analysis, an active investor can estimate bounds on the stock price. In this context, the risk, expected return, and the optimal investment policy of the active investor depend on the current price of the stock relative to the bounds. We consider two types of models, one with reversion from the boundaries, and another in which the investor, in addition to the boundaries, has a belief regarding the risk premium of the stock. We derive optimal 
investment strategies for both classes of models, when the active investor choses between investment in a single stock and a market index. Our results show how an investor who places fundamental boundaries on a stock price will alter his investment strategy. We also demonstrate how the risk and expected return perceived by such an active investor depend on the current stock price.

One extension of our analysis would be to consider multiple stocks. More precisely, in the case of $d$ stocks, we can replace $\sigma B$ in stock $i$ with

$$
\sum_{j=1}^{d} \sigma_{i j} B_{T}^{(j)}, \quad i=1, \ldots, d
$$

for a given $d$-dimensional Brownian Motion $B=\left(B^{(1)}, \ldots, B^{(d)}\right)$. Appropriately choosing $\sigma_{i j}$ 's produces various correlation structures on the stocks. We can also replace $\sigma^{2}$ for stock $i$ with

$$
\Sigma_{i}^{2}=\sum_{j=1}^{d} \sigma_{i j}^{2}
$$

and since we can write

$$
\sum_{j=1}^{d} \sigma_{i j} B_{T}^{(j)}=\Sigma_{i} W_{T}^{(i)}
$$

for some one-dimensional Brownian Motion $W^{(i)}$, we can use the RD model derived for individual stocks. To model a multi-dimensional distribution of the vector $\left(S_{T}^{1}, \ldots, S_{T}^{d}\right)$, a copula approach could be employed. From the theoretical standpoint, it would also be of interest to analyze full equilibrium models in the presence of investors with $\mathrm{RR}$ or $\mathrm{RD}$ beliefs. 


\section{References}

[1] Basak, S. and B. Croitoru, 2000, Equilibrium Mispricing in a Capital Market with Portfolio Constraints, The Review of Financial Studies, 13(3), 715-748.

[2] Black, F. and R. Litterman, 1990, Asset Allocation: Combining Investor Views With Market Equilibrium, Goldman, Sachs 8 Co., Fixed Income Research.

[3] Black, F. and R. Litterman, 1992, Global Portfolio Optimization,Financial Analysts Journal, $28-43$.

[4] Black, F. and M. Scholes, 1973, The Pricing of Options and Corporate Liabilities, Journal of Political Economy, 81:3, 637-654.

[5] Campbell, J.Y., and R.J. Shiller, 2001, Valuation Ratios and the Long-Run Stock Market Outlook: An Update. NBER Working Paper 8221.

[6] Carhart, M.M, 1997, On persistence in mutual fund performance, Journal of Finance, $52,57-82$.

[7] Cochrane, J.H., 2005, Asset Pricing, Revised Edition. Princeton University Press.

[8] Coval, J. D., and T.J. Moskowitz, 1999, Home bias at home: Local equity preference in domestic portfolios, Journal of Finance, 54, 2045-2074.

[9] Coval, J.D., and T. J. Moskowitz, 2001, The geography of investment: Informed trading and asset prices, Journal of Political Economy, 109(4), 811-841.

[10] De Long, J. B., A. Shleifer, L. Summers, and R.J. Waldmann, 1990, Positive feedback investment strategies and destabilizing rational speculation, Journal of Finance 45, 375-395.

[11] Fama, E. F., 1970, Efficient Capital Markets: A Review of Theory and Empirical Work. Journal of Finance, 25, 383-417.

[12] Fama, E. F., 1998, Market Efficiency, Long-Term Returns and Behavioral Finance. Journal of Financial Economics, 49, 283-306.

[13] Fama, E.F. and K.R. French, 1992, The cross section of expected stock returns, Journal of Finance, 47, 427-465.

[14] Fama, E.F. and K.R. French, 1993, Common risk factors in returns on stocks and bonds, Journal of Financial Economics, 33, 3-56.

[15] Fama, E.F. and K.R. French, 1993, Disagreements, tastes and asset prices, Journal of Financial Economics, 83 667-690.

[16] Fama, Eugene F.,1996, Multifactor explanations of asset pricing anomalies, Journal of Finance, 51, 55-84. 
[17] Friedman, M., 1953, The case for flexible exchange rates, in Essays in Positive Economics. University of Chicago Press, Chicago.

[18] Lintner, J., 1965a, The Valuation of Risk Assets and the Selection of Risky Investments in Stock Portfolios and Capital Budgets, Review of Economics and Statistics, 47 (February): 13-37.

[19] Lintner, J., 1965b, Security Prices, Risk and Maximal Gains From Diversification, Journal of Finance, 20 (December): 587 - 615.

[20] Liu, J., and F. A. Longstaff 2004, Losing Money on Arbitrage: Optimal Dynamic Portfolio Choice in Markets with Arbitrage Opportunities, The Review of Financial Studies, 17, 611-641.

[21] Markowitz, H. M., 1952, Portfolio Selection, Journal of Finance 7 (1): 77-91.

[22] Merton, R., 1969, Lifetime portfolio selection under uncertainty: the continuous time case. The Review of Economics and Statistics, 51, 247-257.

[23] Merton, R., 1971, Optimal consumption and portfolio rules in a continuous time model, Journal of Economic Theory, 3, 373-413.

[24] Merton, R. C., 1973, Theory of Rational Option Pricing, Bell Journal of Economics and Management Science, Vol. 4, No. 1, 141-183.

[25] Mossin, J., 1966, Equilibrium in a Capital Asset Market, Econometrica, 35, 768-783.

[26] Penman, S. H., 2007, Financial Statement Analysis and Security Valuation, 3rd edition, McGraw-Hill, New York.

[27] Sharpe, W.L., 1964, Capital Asset Prices: A Theory of Market Equilibrium Under Conditions of Risk, Journal of Finance, 19 (September): 425 - 442.

[28] Sharpe, W.L., 1970, Portfolio Theory and Capital Markets, New York: McGraw-Hill.

[29] Shiller, R., 1984, Stock prices and social dynamics, Brookings Papers on Economic Activity 2, 457-498. 


\section{Table 1}

\section{Optimal allocation in the Range Reversion (RR) case}

The column $\pi^{S}$ measures the optimal proportion of wealth to be invested in the risky security, $\pi^{I}$ measures the optimal proportion of wealth to be invested in the Index, in the Range Reversion Model (RR). The column $\hat{\pi}^{S}$ measures the optimal proportion of wealth to be invested in the risky security, $\hat{\pi}^{I}$ measures the optimal proportion of wealth to be invested in the Index, in the Black-Scholes Model (BSM). $S_{0}$ is the initial price of the security, the range reversion parameters are taken to be $n_{L}=n_{U}=1$. We take $T=1, x=1, \mu=$ $0.08, \sigma=0.3, k=2, r=0.03, \mu_{I}=0.05, \sigma_{I}=0.25, \rho=0.2, \gamma=1$.

PANEL A - Markowitz

\begin{tabular}{ccccc}
\hline & & & & \\
$S_{0}$ & $\pi^{S}$ & $\pi^{I}$ & $\hat{\pi}^{S}$ & $\hat{\pi}^{I}$ \\
\hline & & & & \\
0.595 & 0.932 & 0.074 & 0.457 & 0.176 \\
0.663 & 0.674 & 0.134 & 0.457 & 0.176 \\
0.732 & 0.554 & 0.164 & 0.457 & 0.176 \\
0.801 & 0.498 & 0.175 & 0.457 & 0.176 \\
0.870 & 0.475 & 0.179 & 0.457 & 0.176 \\
0.939 & 0.462 & 0.181 & 0.457 & 0.176 \\
\hline 1.000 & 0.458 & 0.181 & 0.457 & 0.176 \\
\hline 1.008 & 0.456 & 0.183 & 0.457 & 0.176 \\
1.077 & 0.453 & 0.184 & 0.457 & 0.176 \\
1.146 & 0.451 & 0.184 & 0.457 & 0.176 \\
1.215 & 0.447 & 0.189 & 0.457 & 0.176 \\
1.284 & 0.438 & 0.194 & 0.457 & 0.176 \\
1.353 & 0.421 & 0.197 & 0.457 & 0.176 \\
1.422 & 0.396 & 0.207 & 0.457 & 0.176 \\
1.491 & 0.359 & 0.218 & 0.457 & 0.176 \\
1.560 & 0.304 & 0.233 & 0.457 & 0.176 \\
1.629 & 0.225 & 0.252 & 0.457 & 0.176 \\
1.698 & 0.115 & 0.276 & 0.457 & 0.176 \\
1.767 & -0.037 & 0.306 & 0.457 & 0.176 \\
1.836 & -0.223 & 0.340 & 0.457 & 0.176 \\
1.905 & -0.464 & 0.379 & 0.457 & 0.176 \\
1.974 & -0.742 & 0.415 & 0.457 & 0.176 \\
\hline
\end{tabular}

PANEL B - Dynamic

\begin{tabular}{ccccc}
\hline & & & & \\
$S_{0}$ & $\pi^{S}$ & $\pi^{I}$ & $\hat{\pi}^{S}$ & $\hat{\pi}^{I}$ \\
\hline & & & & \\
0.595 & 1.146 & 0.045 & 0.531 & 0.194 \\
0.663 & 0.829 & 0.121 & 0.531 & 0.194 \\
0.732 & 0.659 & 0.162 & 0.531 & 0.194 \\
0.801 & 0.579 & 0.181 & 0.531 & 0.194 \\
0.870 & 0.544 & 0.189 & 0.531 & 0.194 \\
0.939 & 0.531 & 0.193 & 0.531 & 0.194 \\
\hline 1.000 & 0.526 & 0.194 & 0.531 & 0.194 \\
\hline 1.008 & 0.525 & 0.194 & 0.531 & 0.194 \\
1.077 & 0.522 & 0.194 & 0.531 & 0.194 \\
1.146 & 0.518 & 0.196 & 0.531 & 0.194 \\
1.215 & 0.510 & 0.198 & 0.531 & 0.194 \\
1.284 & 0.497 & 0.201 & 0.531 & 0.194 \\
1.353 & 0.475 & 0.206 & 0.531 & 0.194 \\
1.422 & 0.442 & 0.214 & 0.531 & 0.194 \\
1.491 & 0.396 & 0.225 & 0.531 & 0.194 \\
1.560 & 0.333 & 0.240 & 0.531 & 0.194 \\
1.629 & 0.254 & 0.259 & 0.531 & 0.194 \\
1.698 & 0.158 & 0.282 & 0.531 & 0.194 \\
1.767 & 0.047 & 0.309 & 0.531 & 0.194 \\
1.836 & -0.076 & 0.338 & 0.531 & 0.194 \\
1.905 & -0.211 & 0.371 & 0.531 & 0.194 \\
1.974 & -0.349 & 0.404 & 0.531 & 0.194 \\
\hline & & & &
\end{tabular}


Table 2

\section{Optimal allocation to the risky security in the Range Reversion (RR) case} with various range reversion speeds

The column $\pi^{S}$ measures the optimal proportion of wealth to be invested in the risky security in the Range Reversion Model (RR). $S_{0}$ is the initial price of the security. $n_{L}$ and $n_{U}$ are the range reversion parameters. We use $n$ (in paranthesis below) for the values of $n_{L}=n_{U}$. We take $T=1, x=1, \mu=0.08, \sigma=0.3, k=2, r=0.03, \mu_{I}=0.05, \sigma_{I}=0.25, \rho=0.2, \gamma=1$.

\begin{tabular}{ccccccc}
$S_{0}$ & $\pi^{S}(n=0.01)$ & $\pi^{S}(n=0.1)$ & $\pi^{S}(n=1)$ & $\pi^{S}(n=10)$ & $\pi^{S}(n=100)$ & $\pi^{S}(n=1000)$ \\
\hline 0.595 & 0.457 & 0.506 & 0.932 & 1.835 & 1.993 & 1.995 \\
0.663 & 0.453 & 0.476 & 0.674 & 1.287 & 1.623 & 1.738 \\
0.732 & 0.452 & 0.462 & 0.554 & 0.870 & 1.113 & 1.213 \\
0.801 & 0.451 & 0.457 & 0.498 & 0.657 & 0.793 & 0.856 \\
0.870 & 0.451 & 0.453 & 0.475 & 0.554 & 0.628 & 0.664 \\
0.939 & 0.452 & 0.451 & 0.462 & 0.504 & 0.544 & 0.564 \\
\hline 1.000 & 0.451 & 0.452 & 0.458 & 0.479 & 0.500 & 0.509 \\
\hline 1.008 & 0.451 & 0.452 & 0.456 & 0.476 & 0.494 & 0.503 \\
1.077 & 0.451 & 0.450 & 0.453 & 0.459 & 0.456 & 0.453 \\
1.146 & 0.451 & 0.452 & 0.451 & 0.435 & 0.411 & 0.395 \\
1.215 & 0.451 & 0.450 & 0.447 & 0.339 & 0.336 & 0.305 \\
1.284 & 0.451 & 0.450 & 0.438 & 0.340 & 0.217 & 0.156 \\
1.353 & 0.451 & 0.448 & 0.421 & 0.240 & 0.026 & -0.084 \\
1.422 & 0.450 & 0.447 & 0.396 & 0.080 & -0.277 & -0.448 \\
1.491 & 0.449 & 0.443 & 0.359 & -0.171 & -0.715 & -0.972 \\
1.560 & 0.450 & 0.439 & 0.304 & -0.532 & -1.308 & -1.635 \\
1.629 & 0.448 & 0.433 & 0.225 & -1.020 & -2.003 & -2.360 \\
1.698 & 0.449 & 0.423 & 0.115 & -1.633 & -2.684 & -2.984 \\
1.767 & 0.447 & 0.415 & -0.037 & -2.255 & -3.194 & -3.388 \\
1.836 & 0.446 & 0.401 & -0.223 & -2.846 & -3.487 & -3.545 \\
1.905 & 0.445 & 0.386 & -0.464 & -3.246 & -3.552 & -3.523 \\
1.974 & 0.443 & 0.367 & -0.742 & -3.443 & -3.475 & -3.394 \\
\hline
\end{tabular}




\section{Table 3}

\section{Risk Premia for BSMCT Models}

Column $\theta$ measures the risk premium in the corresponding case of the BSMCT model. $S_{0}$ is the initial price of the security. We take $T=1, x=1, \mu=0.08, \sigma=0.3, k=2, r=$ $0.03, \mu_{I}=0.05, \sigma_{I}=0.25, \rho=0.2, n_{L}=n_{U}=1, \gamma=1$.

\begin{tabular}{cccc}
$S_{0}$ & $\theta$ (m-fixed) & $\theta$ (m-floating) & $\theta(L=0, U=\infty)$ \\
\hline 0.595 & 3.197 & 2.122 & 1.900 \\
0.663 & 2.036 & 1.027 & 1.534 \\
0.732 & 1.476 & 0.649 & 1.205 \\
0.801 & 1.064 & 0.448 & 0.905 \\
0.870 & 0.724 & 0.325 & 0.629 \\
0.939 & 0.425 & 0.242 & 0.375 \\
\hline 1.000 & 0.185 & 0.185 & 0.167 \\
\hline 1.008 & 0.154 & 0.178 & 0.139 \\
1.077 & -0.098 & 0.121 & -0.082 \\
1.146 & -0.336 & 0.064 & -0.288 \\
1.215 & -0.565 & 0.000 & -0.483 \\
1.284 & -0.788 & -0.074 & -0.667 \\
1.353 & -1.009 & -0.162 & -0.842 \\
1.422 & -1.232 & -0.267 & -1.007 \\
1.491 & -1.461 & -0.393 & -1.165 \\
1.560 & -1.701 & -0.546 & -1.316 \\
1.629 & -1.958 & -0.733 & -1.460 \\
1.698 & -2.249 & -0.970 & -1.598 \\
1.767 & -2.598 & -1.286 & -1.731 \\
1.836 & -3.084 & -1.768 & -1.859 \\
1.905 & -4.236 & -3.041 & -1.981 \\
1.974 & -5.912 & -5.346 & -2.100 \\
\hline & & &
\end{tabular}




\section{Table 4}

Expected Returns and Variances of Returns for all four models: RR and three BSMCT models

The columns in Panel A represent the Expected Returns in the corresponding case and in Panel B they represent the Variances of Returns in the corresponding case. We take $T=1, x=1, \mu=0.08, \sigma=0.3, k=2, r=0.03, \mu_{I}=0.05, \sigma_{I}=0.25, \rho=0.2, n_{L}=n_{U}=$ $1, \gamma=1$.

PANEL A

\begin{tabular}{ccccc}
$S_{0}$ & RR & m-fix & m-flo & $L=0, U=\infty$ \\
\hline & & & & \\
0.595 & 1.123 & 1.822 & 1.312 & 1.822 \\
0.663 & 1.101 & 1.633 & 1.238 & 1.633 \\
0.732 & 1.091 & 1.479 & 1.185 & 1.479 \\
0.801 & 1.087 & 1.352 & 1.148 & 1.352 \\
0.870 & 1.085 & 1.245 & 1.120 & 1.245 \\
0.939 & 1.084 & 1.153 & 1.099 & 1.153 \\
\hline 1.000 & 1.083 & 1.083 & 1.083 & 1.083 \\
\hline 1.008 & 1.083 & 1.074 & 1.081 & 1.074 \\
1.077 & 1.082 & 1.006 & 1.065 & 1.006 \\
1.146 & 1.081 & 0.945 & 1.048 & 0.945 \\
1.215 & 1.079 & 0.891 & 1.031 & 0.891 \\
1.284 & 1.077 & 0.844 & 1.013 & 0.844 \\
1.353 & 1.074 & 0.801 & 0.994 & 0.801 \\
1.422 & 1.070 & 0.762 & 0.974 & 0.762 \\
1.491 & 1.065 & 0.727 & 0.954 & 0.727 \\
1.560 & 1.059 & 0.694 & 0.932 & 0.694 \\
1.629 & 1.051 & 0.665 & 0.911 & 0.665 \\
1.698 & 1.043 & 0.638 & 0.890 & 0.638 \\
1.767 & 1.032 & 0.613 & 0.869 & 0.613 \\
1.836 & 1.021 & 0.590 & 0.848 & 0.590 \\
1.905 & 1.008 & 0.569 & 0.828 & 0.569 \\
1.974 & 0.994 & 0.549 & 0.808 & 0.549 \\
\hline
\end{tabular}

PANEL B

\begin{tabular}{lllll}
$S_{0}$ & $\mathrm{RR}$ & $\mathrm{m}$-fix & $\mathrm{m}$-flo & $L=0, U=\infty$ \\
\hline
\end{tabular}

\begin{tabular}{lllll}
0.595 & 0.093 & 0.237 & 0.073 & 0.313 \\
0.663 & 0.100 & 0.190 & 0.079 & 0.251 \\
0.732 & 0.105 & 0.156 & 0.084 & 0.206 \\
0.801 & 0.107 & 0.131 & 0.087 & 0.172 \\
0.870 & 0.108 & 0.111 & 0.088 & 0.146 \\
0.939 & 0.109 & 0.095 & 0.087 & 0.125 \\
\hline 1.000 & 0.108 & 0.084 & 0.084 & 0.111 \\
\hline 1.008 & 0.108 & 0.082 & 0.083 & 0.109 \\
1.077 & 0.107 & 0.072 & 0.078 & 0.095 \\
1.146 & 0.106 & 0.064 & 0.072 & 0.084 \\
1.215 & 0.103 & 0.057 & 0.066 & 0.075 \\
1.284 & 0.100 & 0.051 & 0.059 & 0.067 \\
1.353 & 0.097 & 0.046 & 0.052 & 0.060 \\
1.422 & 0.093 & 0.041 & 0.046 & 0.055 \\
1.491 & 0.088 & 0.038 & 0.041 & 0.050 \\
1.560 & 0.083 & 0.034 & 0.036 & 0.045 \\
1.629 & 0.078 & 0.032 & 0.032 & 0.042 \\
1.698 & 0.073 & 0.029 & 0.028 & 0.038 \\
1.767 & 0.069 & 0.027 & 0.024 & 0.035 \\
1.836 & 0.064 & 0.025 & 0.022 & 0.033 \\
1.905 & 0.060 & 0.023 & 0.019 & 0.030 \\
1.974 & 0.055 & 0.022 & 0.017 & 0.028 \\
\hline
\end{tabular}




\section{Table 5}

\section{Betas and Instantaneous betas for the four models: RR and three BSMCT}

models

The columns in Panel A represent the Betas, and in Panel B they represent the Instantaneous

Betas in the corresponding case. We take $T=1, x=1, \mu=0.08, \sigma=0.3, k=2, r=$ $0.03, \mu_{I}=0.05, \sigma_{I}=0.25, \rho=0.2, n_{L}=n_{U}=1, \gamma=1$.

PANEL A

\begin{tabular}{ccccc}
\hline$S_{0}$ & RR & m-fix & m-flo & $L=0, U=\infty$ \\
\hline & & & & \\
0.595 & 0.216 & 0.354 & 0.189 & 0.406 \\
0.663 & 0.227 & 0.318 & 0.197 & 0.364 \\
0.732 & 0.228 & 0.288 & 0.206 & 0.329 \\
0.801 & 0.234 & 0.263 & 0.211 & 0.301 \\
0.870 & 0.239 & 0.243 & 0.214 & 0.278 \\
0.939 & 0.241 & 0.224 & 0.213 & 0.257 \\
\hline 1.000 & 0.243 & 0.210 & 0.211 & 0.242 \\
\hline 1.008 & 0.241 & 0.209 & 0.210 & 0.240 \\
1.077 & 0.240 & 0.196 & 0.204 & 0.225 \\
1.146 & 0.241 & 0.184 & 0.196 & 0.211 \\
1.215 & 0.233 & 0.173 & 0.188 & 0.198 \\
1.284 & 0.288 & 0.165 & 0.177 & 0.188 \\
1.353 & 0.230 & 0.156 & 0.168 & 0.179 \\
1.422 & 0.223 & 0.149 & 0.158 & 0.170 \\
1.491 & 0.218 & 0.141 & 0.148 & 0.162 \\
1.560 & 0.211 & 0.135 & 0.138 & 0.155 \\
1.629 & 0.206 & 0.130 & 0.129 & 0.148 \\
1.698 & 0.198 & 0.124 & 0.121 & 0.142 \\
1.767 & 0.198 & 0.119 & 0.113 & 0.137 \\
1.836 & 0.185 & 0.115 & 0.105 & 0.132 \\
1.905 & 0.180 & 0.111 & 0.099 & 0.127 \\
1.974 & 0.167 & 0.107 & 0.093 & 0.122 \\
\hline & & & &
\end{tabular}

PANEL B

\begin{tabular}{cccc}
$S_{0}$ & m-fix & m-flo & $L=0, U=\infty$ \\
\hline & & & \\
0.595 & 0.039 & 0.034 & 0.240 \\
0.663 & 0.120 & 0.109 & 0.240 \\
0.732 & 0.162 & 0.156 & 0.240 \\
0.801 & 0.186 & 0.180 & 0.240 \\
0.870 & 0.200 & 0.197 & 0.240 \\
0.939 & 0.207 & 0.207 & 0.240 \\
\hline 1.000 & 0.210 & 0.210 & 0.240 \\
\hline 1.008 & 0.210 & 0.210 & 0.240 \\
1.077 & 0.210 & 0.209 & 0.240 \\
1.146 & 0.206 & 0.205 & 0.240 \\
1.215 & 0.201 & 0.197 & 0.240 \\
1.284 & 0.193 & 0.187 & 0.240 \\
1.353 & 0.183 & 0.176 & 0.240 \\
1.422 & 0.172 & 0.162 & 0.240 \\
1.491 & 0.158 & 0.147 & 0.240 \\
1.560 & 0.142 & 0.131 & 0.240 \\
1.629 & 0.124 & 0.112 & 0.240 \\
1.698 & 0.103 & 0.092 & 0.240 \\
1.767 & 0.079 & 0.069 & 0.240 \\
1.836 & 0.049 & 0.043 & 0.240 \\
1.905 & 0.010 & 0.008 & 0.240 \\
1.974 & 0.000 & 0.000 & 0.240 \\
\hline
\end{tabular}




\section{Table 6}

\section{Markowitz Portfolios in BSMCT model with $m$-fixed and $m$-floating.}

The columns in Panel A represent weights on the stock and the index in the case of BSMCT and BSM models, in which $\mathrm{m}$ is fixed. The columns in Panel $\mathrm{B}$ represent weights on the stock and the index in the case of BSMCT and BSM models, in which $\mathrm{m}$ is floating. We use $T=1, x=1, \mu=0.08, \sigma=0.3, k=2, r=0.03, \mu_{I}=0.05, \sigma_{I}=0.25, \rho=0.2, n_{L}=n_{U}=$ $1, \gamma=1$.

PANEL A

\begin{tabular}{ccccc}
\hline & & & & \\
$S_{0}$ & $\pi^{S}$ & $\pi^{I}$ & $\hat{\pi}^{S}$ & $\hat{\pi}^{I}$ \\
\hline & & & & \\
0.595 & 0.957 & -0.258 & 0.616 & 0.037 \\
0.663 & 1.138 & -0.260 & 0.616 & 0.037 \\
0.732 & 1.313 & -0.248 & 0.616 & 0.037 \\
0.801 & 1.439 & -0.212 & 0.616 & 0.037 \\
0.870 & 1.429 & -0.135 & 0.616 & 0.037 \\
0.939 & 1.150 & 0.002 & 0.616 & 0.037 \\
\hline 1.000 & 0.598 & 0.165 & 0.616 & 0.037 \\
\hline 1.008 & 0.502 & 0.188 & 0.616 & 0.037 \\
1.077 & -0.420 & 0.378 & 0.616 & 0.037 \\
1.146 & -1.318 & 0.508 & 0.616 & 0.037 \\
1.215 & -1.953 & 0.557 & 0.616 & 0.037 \\
1.284 & -2.295 & 0.550 & 0.616 & 0.037 \\
1.353 & -2.435 & 0.515 & 0.616 & 0.037 \\
1.422 & -2.457 & 0.470 & 0.616 & 0.037 \\
1.491 & -2.420 & 0.425 & 0.616 & 0.037 \\
1.560 & -2.357 & 0.386 & 0.616 & 0.037 \\
1.629 & -2.284 & 0.351 & 0.616 & 0.037 \\
1.698 & -2.210 & 0.320 & 0.616 & 0.037 \\
1.767 & -2.139 & 0.294 & 0.616 & 0.0373 \\
1.836 & -2.073 & 0.271 & 0.616 & 0.037 \\
1.905 & -2.012 & 0.251 & 0.616 & 0.037 \\
1.974 & -1.956 & 0.233 & 0.616 & 0.037 \\
\hline & & & &
\end{tabular}

PANEL B

\begin{tabular}{ccccc}
$S_{0}$ & $\pi^{S}$ & $\pi^{I}$ & $\hat{\pi}^{S}$ & $\hat{\pi}^{I}$ \\
\hline & & & & \\
0.595 & 1.934 & -0.222 & 0.616 & 0.037 \\
0.663 & 1.779 & -0.156 & 0.616 & 0.037 \\
0.732 & 1.496 & -0.074 & 0.616 & 0.037 \\
0.801 & 1.119 & 0.007 & 0.616 & 0.037 \\
0.870 & 0.952 & 0.072 & 0.616 & 0.037 \\
0.939 & 0.751 & 0.125 & 0.616 & 0.037 \\
\hline 1.000 & 0.598 & 0.165 & 0.616 & 0.037 \\
\hline 1.008 & 0.579 & 0.170 & 0.616 & 0.037 \\
1.077 & 0.402 & 0.214 & 0.616 & 0.037 \\
1.146 & 0.202 & 0.259 & 0.616 & 0.037 \\
1.215 & -0.050 & 0.308 & 0.616 & 0.037 \\
1.284 & -0.381 & 0.364 & 0.616 & 0.037 \\
1.353 & -0.787 & 0.422 & 0.616 & 0.037 \\
1.422 & -1.271 & 0.477 & 0.616 & 0.037 \\
1.491 & -1.796 & 0.523 & 0.616 & 0.037 \\
1.560 & -2.316 & 0.550 & 0.616 & 0.037 \\
1.629 & -2.767 & 0.557 & 0.616 & 0.037 \\
1.698 & -3.114 & 0.545 & 0.616 & 0.037 \\
1.767 & -3.345 & 0.519 & 0.616 & 0.037 \\
1.836 & -3.463 & 0.478 & 0.616 & 0.037 \\
1.905 & -3.497 & 0.437 & 0.616 & 0.037 \\
1.974 & -3.470 & 0.396 & 0.616 & 0.037 \\
\hline & & & &
\end{tabular}




\section{Table 6}

Markowitz Portfolios in BSMCT model with $L=0, U=\infty$

The Panel C represents weights on the stock and the index in the case of BSMCT and BSM models, in which $L=0, U=\infty$. We use $T=1, x=1, \mu=0.08, \sigma=0.3, k=2, r=$ $0.03, \mu_{I}=0.05, \sigma_{I}=0.25, \rho=0.2$.

\begin{tabular}{ccccc}
\multicolumn{5}{c}{ PANEL C } \\
\hline$S_{0}$ & $\pi^{S}$ & $\pi^{I}$ & $\hat{\pi}^{S}$ & $\hat{\pi}^{I}$ \\
\hline & & & & \\
0.595 & 0.881 & -0.259 & 0.449 & 0.184 \\
0.663 & 1.027 & -0.252 & 0.449 & 0.184 \\
0.732 & 1.154 & -0.229 & 0.449 & 0.184 \\
0.801 & 1.221 & -0.180 & 0.449 & 0.184 \\
0.870 & 1.162 & -0.094 & 0.449 & 0.184 \\
0.939 & 0.895 & 0.039 & 0.449 & 0.184 \\
\hline 1.000 & 0.451 & 0.184 & 0.449 & 0.184 \\
\hline 1.008 & 0.375 & 0.205 & 0.449 & 0.184 \\
1.077 & -0.325 & 0.369 & 0.449 & 0.184 \\
1.146 & -1.032 & 0.490 & 0.449 & 0.184 \\
1.215 & -1.588 & 0.548 & 0.449 & 0.184 \\
1.284 & -1.940 & 0.557 & 0.449 & 0.184 \\
1.353 & -2.130 & 0.535 & 0.449 & 0.184 \\
1.422 & -2.208 & 0.500 & 0.449 & 0.184 \\
1.491 & -2.221 & 0.461 & 0.449 & 0.184 \\
1.560 & -2.198 & 0.423 & 0.449 & 0.184 \\
1.629 & -2.156 & 0.388 & 0.449 & 0.184 \\
1.698 & -2.106 & 0.357 & 0.449 & 0.184 \\
1.767 & -2.054 & 0.329 & 0.449 & 0.184 \\
1.836 & -2.002 & 0.305 & 0.449 & 0.184 \\
1.905 & -1.952 & 0.283 & 0.449 & 0.184 \\
1.974 & -1.905 & 0.265 & 0.449 & 0.184 \\
1.974 & -3.470 & 0.396 & 0.449 & 0.184 \\
\hline & & & & \\
\hline
\end{tabular}


Table 7

\section{Optimal allocation to stocks in all four models}

The columns represent the optimal allocation to the risky security in all 4 Markowitz cases: $\mathrm{RR}$ model, BSMCT case when $\mathrm{m}$ is fixed, BSMCT case when $\mathrm{m}$ is floating, and BSMCT case when $L=0, U=\infty$. We take $T=1, x=1, \mu=0.08, \sigma=0.3, k=2, r=0.03, \mu_{I}=$ $0.05, \sigma_{I}=0.25, \rho=0.2, n_{L}=n_{U}=1, \gamma=1$.

\begin{tabular}{ccccc}
\hline$S_{0}$ & RR & m-fix & m-flo & $L=0, U=\infty$ \\
\hline & & & & \\
0.595 & 0.932 & 0.957 & 1.934 & 0.881 \\
0.663 & 0.674 & 1.138 & 1.779 & 1.027 \\
0.732 & 0.554 & 1.313 & 1.496 & 1.154 \\
0.801 & 0.498 & 1.439 & 1.199 & 1.221 \\
0.870 & 0.475 & 1.429 & 0.952 & 1.162 \\
0.939 & 0.462 & 1.150 & 0.751 & 0.895 \\
\hline 1.000 & 0.458 & 0.598 & 0.598 & 0.451 \\
\hline 1.008 & 0.456 & 0.502 & 0.579 & 0.375 \\
1.077 & 0.453 & -0.420 & 0.402 & -0.325 \\
1.146 & 0.451 & -1.318 & 0.202 & -1.032 \\
1.215 & 0.447 & -1.953 & -0.050 & -1.588 \\
1.284 & 0.438 & -2.295 & -0.381 & -1.940 \\
1.353 & 0.421 & -2.435 & -0.787 & -2.130 \\
1.422 & 0.396 & -2.457 & -1.271 & -2.208 \\
1.491 & 0.359 & -2.420 & -1.796 & -2.221 \\
1.560 & 0.304 & -2.357 & -2.316 & -2.198 \\
1.629 & 0.225 & -2.284 & -2.767 & -2.156 \\
1.698 & 0.115 & -2.210 & -3.114 & -2.106 \\
1.767 & -0.037 & -2.139 & -3.345 & -2.054 \\
1.836 & -0.223 & -2.073 & -3.463 & -2.002 \\
1.905 & -0.464 & -2.012 & -3.497 & -1.952 \\
1.974 & -0.742 & -1.956 & -3.470 & -1.905 \\
\hline
\end{tabular}




\section{Table 8}

\section{Certainty Equivalents for all models}

The columns represent the Certainty Equivalents for all models in all 4 Markowitz cases: $\mathrm{RR}$ model, BSMCT case when $\mathrm{m}$ is fixed, BSMCT case when $\mathrm{m}$ is floating, and BSMCT case when $L=0, U=\infty$. We take $T=1, x=1, \mu=0.08, \sigma=0.3, k=2, r=0.03, \mu_{I}=$ $0.05, \sigma_{I}=0.25, \rho=0.2, n_{L}=n_{U}=1, \gamma=1$.

In cases (i) we compute the following Certainty Equivalence ratios: $\frac{C E\left(S^{R R}, \delta_{S}^{B S M}, \delta_{I}^{B S M}\right)}{C E\left(S^{R R}, \delta_{S}^{R R}, \delta_{I}^{R R}\right)}$, and in cases (ii) we compute the following Certainty Equivalence ratios: $\frac{C E\left(S^{B S M}, \delta_{S}^{R R}, \delta_{I}^{R R}\right)}{C E\left(S^{B S M}, \delta_{S}^{B S M}, \delta_{I}^{B S M}\right)}$

\begin{tabular}{ccccccccc}
\hline$S_{0}$ & RR-i & RR-ii & m-fix-i & m-fix-ii & m-flo-i & m-flo-ii & $L=0, U=\infty$-i & $L=0, U=\infty$-ii \\
\hline & & & & & & & & \\
0.595 & 0.754 & 0.161 & 0.828 & 0.486 & 0.494 & -3.107 & 0.703 & 0.006 \\
0.663 & 0.909 & 0.825 & 0.740 & 0.151 & 0.551 & -2.219 & 0.634 & -0.484 \\
0.732 & 0.974 & 0.965 & 0.676 & -0.308 & 0.645 & -0.868 & 0.589 & -1.025 \\
0.801 & 0.994 & 0.994 & 0.643 & -0.707 & 0.761 & 0.174 & 0.578 & -1.330 \\
0.870 & 0.999 & 0.999 & 0.662 & -0.624 & 0.871 & 0.733 & 0.617 & -0.959 \\
0.939 & 1.000 & 1.000 & 0.782 & 0.306 & 0.952 & 0.976 & 0.760 & 0.234 \\
\hline 1.000 & 1.000 & 1.000 & 0.969 & 1.032 & 0.969 & 1.032 & 1.000 & 1.000 \\
\hline 1.008 & 1.000 & 1.000 & 0.918 & 1.010 & 0.964 & 1.031 & 0.973 & 0.979 \\
1.077 & 1.000 & 1.000 & -3.034 & -1.400 & 0.747 & 0.940 & -2.396 & -1.278 \\
1.146 & 1.000 & 1.000 & -0.996 & -7.197 & -0.436 & 0.657 & -0.888 & -7.040 \\
1.215 & 0.999 & 0.999 & -0.620 & -12.953 & -3.460 & 0.036 & -0.548 & -13.621 \\
1.284 & 0.998 & 0.998 & -0.491 & -16.538 & -2.937 & -1.218 & -0.424 & -18.561 \\
1.353 & 0.994 & 0.995 & -0.434 & -18.114 & -1.660 & -3.397 & -0.366 & -21.465 \\
1.422 & 0.983 & 0.986 & -0.407 & -18.413 & -1.019 & -6.828 & -0.336 & -22.744 \\
1.491 & 0.951 & 0.963 & -0.395 & -18.054 & -0.698 & -11.437 & -0.320 & -23.005 \\
1.560 & 0.860 & 0.912 & -0.390 & -17.398 & -0.520 & -16.759 & -0.312 & -22.693 \\
1.629 & 0.594 & 0.798 & -0.389 & -16.645 & -0.417 & -21.897 & -0.307 & -22.090 \\
1.698 & -0.138 & 0.564 & -0.390 & -15.887 & -0.354 & -26.137 & -0.306 & -21.368 \\
1.767 & -1.475 & 0.094 & -0.393 & -15.167 & -0.314 & -29.089 & -0.305 & -20.612 \\
1.836 & -2.010 & -0.708 & -0.397 & -14.507 & -0.290 & -30.671 & -0.306 & -19.863 \\
1.905 & -1.592 & -2.111 & -0.401 & -13.903 & -0.275 & -31.191 & -0.308 & -19.151 \\
1.974 & -1.140 & -4.204 & -0.406 & -13.356 & -0.266 & -30.909 & -0.310 & -18.490 \\
\hline
\end{tabular}

